\title{
Overcoming sociocultural barriers to clinical breast examinations in South Asian immigrant women living in Canada
}

\section{Sasi Shanmugarajah and C. Geeth Gunawardana}

\section{University of Toronto}

On average, 1 in 9 women are expected to develop breast cancer in their lifetime; making it the most common type of cancer (next to non-melanoma skin cancer) in women ${ }^{1}$. Although the mortality rate from breast cancer is $15 \%$, it has declined by over $25 \%$ since $1986^{2}$. This improvement in survival rate can be attributed to the development of more effective screening methods, an increase in screening participation, and advances in clinical breast examinations (CBE).

Despite this progress, current population health research reveals several barriers that influence participation in breast cancer screening programs. These barriers include the fear of pain and embarrassment, the concerns over the use of radiation, and the socio-economic status of the women ${ }^{3}$. One of the most significant barriers identified by Hanson et al. (2009) was whether or not a participant was a member of an ethnic minority. In 2006, visible minorities made up onesixth of the total population of Canada, with those of South Asian origin representing the largest ethnic minority ${ }^{4}$. Immigrant women of South Asian origin show significantly lower breast cancer screening rates than Canadian-born women $^{5}$. Studies show that information about breast cancer and screening are reaching this group but having less of an impact $^{3,6}$. In order to understand why screening rates are lower in this population, we need to understand the sociocultural characteristics of immigrants of South Asian origin.

South Asia comprises of several countries including India, Pakistan, Sri Lanka, and Bangladesh. Each nation is home to a variety of ethnic groups differentiated by religion, language, and social practices. Yet, within this great diversity there are core beliefs and practices that are shared amongst these groups. The work of Bottorff et al. (1998) suggests that these beliefs and practices play a significant role in forming barriers to breast health practices among South Asian women in Canada $^{7}$. The following is a list of some of the regional commonalities that influence health and health care: practices among South Asian women in Canada ${ }^{7}$ The following is a list of some of the regional commonalities that influence health and health care:
1. Standards of modesty - Touching oneself or being touched by someone else (a common occurrence in $\mathrm{CBE}$ ) is considered taboo.

2. Gender role - The needs of the family unit outweigh the needs of the individual. This leads to women deprioritizing their personal health issues in favour of their husband and/or their children.

3. Superstition - Some do not want to utter the word "cancer", think about cancer, or be associated with cancer screening for the fear that one would be tempting fate.

4. Spiritual Beliefs - Believing that if it is in one's karma, a concept prevalent in South Asian culture, to develop cancer, then screening will not prevent the consequences as it is unavoidable.

5. Physician on a pedestal - The "doctor is always right" philosophy prevails in South Asian culture, and if a CBE is not suggested (for example when a person is considered low risk due to age or genetics), then South Asian women may feel uncomfortable going against the doctor's advice by asking for a CBE.

6. Family's honor and reputation - Arranged marriage is practiced in much of South Asia. A family's marriage potential is assessed by factors such as hereditary traits and health, where good health is believed to be a sign of a good 'pedigree'. Thus, women fear that the results of a $\mathrm{CBE}$ may tarnish the family's reputation.

Although some acculturation to Canadian customs occurs among new immigrants, many still retain traditional preferences, including views on family and religion - the core beliefs that traditional preferences, including views on family and religion - the core beliefs that support and influence the cultural barriers described above to current screening programs $^{8}$. Organized screening programs need to circumvent these cultural undertones to overcome the breast health inequalities seen in Canada's South Asian minority.

An effective method to reach this vulnerable group is to tailor health promotion around socio-cultural characteristics. Ahmad et al. (2004) used such an approach on a cohort of South Asian women that showed low compliancy to CBE (less than one-third $)^{9}$. The investigators tailored a health promotion intervention that tackled the barriers mentioned previously by the following methods: 
1. Demonstrating that screening not only benefited the individual but also improved the quality of family life.

2. Encouraging women to discuss breast health with family, relatives, and health care providers

3. Emphasizing the availability of female health personnel to overcome modesty and apprehension.

In the follow-up to the intervention, the cohort showed significant improvement in breast cancer knowledge, an increase in self-efficacy to discuss breast health, and most importantly, an increase in participation in CBE. The success of Ahmad et al. (2004) supports the hypothesis that socioculturally tailored health interventions can improve breast health practices in this vulnerable group ${ }^{9}$. In constructing future intervention policies, we suggest the following tailored approach to the South Asian community, some of which have been highlighted by others ${ }^{3,9}$ :

1. Direct health information not just at women, but also at their family and community, which can play important roles in overcoming the stigma of cancer in the South Asian population. These two groups can be reached through discourse on ethnic TV and radio programs. Health information articles in ethnic newspapers can also ensure that the message reaches not only women but also their families, thus promoting familial responsibility in health care. It is also important that South Asian leaders in the community, male and female, take the initiative to actively educate the population through community events.

2. Provide socio-cultural sensitive information on breast cancer that highlights the impact of breast cancer on the community and the significance and benefits of CBE. This information should also highlight the responsibility of individuals and their families to take ownership of their own health care needs, even going so far as to challenge doctor recommendations if they feel there is a real problem.

3. Address the South Asian women's need for privacy during CBE by educating physicians about this stigma. For example, doctors could use more discrete questioning practices with patients when discussing the possibility having cancer to avoid unnecessary stress. Another suggestion is to provide videos demonstrating proper selfexamination techniques that can be watched in the privacy of a home. Furthermore, physicians with an understanding of the significance of "family honour" in South Asian culture can also provide more meaningful reassurance of examination

confidentiality.

4. Allow female relatives or friends to attend the patient's CBE. This can go a long way in providing South Asian patients with the support they need to overcome their modesty concerns with CBE.
In summary, tailored socio-cultural health promotion and interventions methods would be more effective in ensuring breast cancer messages are understood by a South Asian immigrant audience, as the information is directly relevant to the community, commanding their attention, and reducing defensiveness to the breast cancer issue. In time, tailored intervention programs to South Asians, as well as other ethnic groups, can dramatically improve early detection of breast cancer in ethnic communities which may lead to lower breast cancer mortality rates.

\section{References}

1. Canadian Cancer Statistics 2010. (Canadian Cancer Society, 2010).

2. Canadian Cancer Statistics 2007. (Canadian Cancer Society, 2007).

3. Hanson, K., Montgomery, P., Bakker, D. \& Conlon, M. Factors influencing mammography participation in Canada: an integrative review of the literature. Curr Oncol 16, 65-75 (2009).

4. 2006 Census. (Statistics Canada, 2006).

5. Ahmad, F. \& Stewart, D.E. Predictors of clinical breast examination among South Asian immigrant women. J Immigr Health 6, 119-126 (2004)

6. Sun, Z., et al. Breast cancer screening among Asian immigrant women in Canada. Cancer Epidemiol 34, 73-78.

7. Bottorff, J.L., et al. Beliefs related to breast health practices: the perceptions of South Asian women living in Canada. Soc Sci Med 47, 2075-2085 (1998).

8. Brotto, L.A., Chou, A.Y., Singh, T. \& Woo, J.S. Reproductive health practices among Indian, Indo-Canadian, Canadian East Asian, and Euro-Canadian women: the role of acculturation. $J$ Obstet Gynaecol Can 30, 229-238 (2008).

9. Ahmad, F., Cameron, J.I. \& Stewart, D.E. A tailored intervention to promote breast cancer screening among South Asian immigrant women. Soc Sci Med 60, 575-586 (2005).

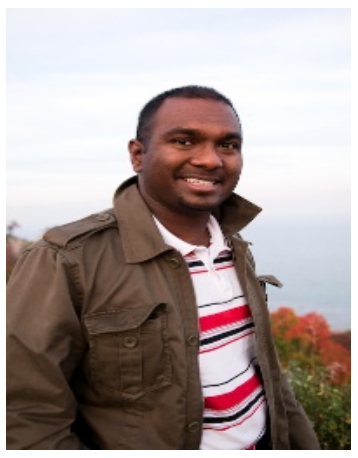

Author Profiles

Sasi Shanmugarajah completed his undergraduate in Human Biology in 1999 at the University of Toronto, returned for an undergrad in Computer Engineering in 2003, and went back a third time for his MBA in 2010. He currently works in the technology industry and has an avid interest in medicine and the health sciences.

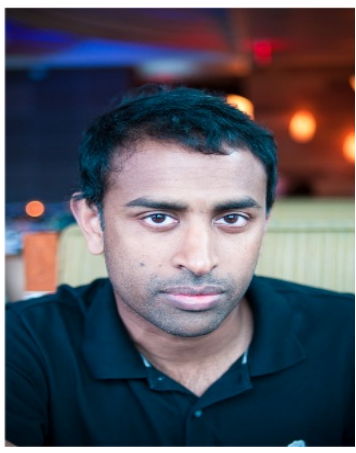

Geeth Gunawardana graduated with a $\mathrm{PhD}$ from the Department of Laboratory Medicine \& Pathobiology at the University of Toronto. His doctoral work focused on early detection markers of ovarian cancer. His research interests include diagnostic markers, tumour immunology, drug development, and health interventions methods. 